EFI-93-21

\title{
The Light Cone in String Theory
}

\author{
Emil Martinec* \\ Enrico Fermi Inst. and Dept. of Physics \\ University of Chicago, Chicago, IL 60637
}

The causal boundary of string propagation - defined as the hypersurface in loop space bordering the timelike(spacelike) domains in which two successive measurements of the string field do(do not) interfere with one another - is argued to be

$$
0=\int d \sigma(\delta X(\sigma))^{2}=\sum_{\ell=-\infty}^{\infty} \delta x_{-\ell}^{\mu} \delta x_{\mu \ell}
$$

Some possible consequences are discussed.

$4 / 93$

* Supported in part by Dept. of Energy grant DEFG02-90ER-40560. 


\section{Introduction}

In particle field theory, causality is ensured by the fact that a) the commutator of two free fields vanishes in the spacelike region, and b) the interactions are local. Thus, at least in perturbation theory, no two measurements of the field at spacelike separated points can interfere with one another. Surprisingly enough, the analogous question in string theory has not yet been addressed. Here we will calculate the string field commutator in flat spacetime in the light-front gauge. The commutator will indeed vanish outside some hypersurface in loop space. This hypersurface is not simply related to the light cone of the underlying point manifold. We then make an ansatz concerning the covariant generalization of the result, and ask whether causality in string field theory may be quite different than in particle field theory.

\subsection{Light-Front Gauge}

As a warm-up exercise, let us review the computation of the particle-field commutator. We will employ light-front quantization, since in the string case one has a known operator formalism only in light-front gauge. A scalar field is decomposed as

$$
\phi\left(x^{+}, x^{-}, \vec{x}\right)=\int_{-\infty}^{\infty} \frac{d \vec{p}}{(2 \pi)^{d-2}} \int_{0}^{\infty} \frac{d p^{+}}{2 p^{+}}\left(\mathbf{a}_{p^{+}, \vec{p}} e^{\left[i\left(p^{+} x^{-}+p^{-} x^{+}+\vec{p} \cdot \vec{x}\right)\right]}+\text { h.c. }\right),
$$

with $p^{-}=\left(\vec{p}^{2}+m^{2}\right) / 2 p^{+}$, and the particle creation/annihilation operators $\mathbf{a}_{p^{+}, \vec{p}}$ satisfying

$$
\left[\mathbf{a}_{p^{+}, \vec{p}}, \mathbf{a}_{p^{+^{\prime}}, \vec{p}^{\prime}}^{\dagger}\right]=p^{+} \cdot 2 \pi \delta_{p^{+}, p^{+^{\prime}}} \cdot(2 \pi)^{d-2} \delta_{\vec{p}, \vec{p}^{\prime}} .
$$

One finds the commutator

$$
\begin{gathered}
{\left[\phi\left(x^{+}, x^{-}, \vec{x}\right), \phi\left(x^{+^{\prime}}, x^{-^{\prime}}, \vec{x}^{\prime}\right)\right]=\int_{0}^{\infty} \frac{d p^{+}}{2 p^{+}}\left(\mathbf{K}_{p^{+}}^{0}\left(\delta x^{+}, \delta x^{-}, \delta \vec{x}\right)-\mathbf{K}_{p^{+}}^{0}\left(-\delta x^{+},-\delta x^{-},-\delta \vec{x}\right)\right)} \\
\mathbf{K}_{p^{+}}^{0}=\left(\frac{p^{+}}{2 \pi \delta x^{+}}\right)^{(d-2) / 2} \exp i\left[-p^{+} \delta x^{-}+\frac{m^{2}}{2 p^{+}} \delta x^{+}+\frac{p^{+}}{2 \delta x^{+}}(\delta \vec{x})^{2}\right]
\end{gathered}
$$

The convergence properties of the $p^{+}$integral depend crucially on the sign of $(\delta x)^{2}=$ $2 \delta x^{+} \delta x^{-}-\delta \vec{x}^{2}$. For $(\delta x)^{2}<0$, the integration contour can be rotated onto the positive imaginary axis for the first term in the commutator, and onto the negative imaginary axis for the second; both terms are equal and the difference vanishes. For $(\delta x)^{2}>0$, the rotation cannot be made so that the integral converges both at $p^{+} \rightarrow 0$ and $p^{+} \rightarrow \infty$; the two terms in the commutator do not cancel and the measurements of the field interfere. 
Having completed the exercise, the generalization to string theory is straightforward. We work in light-front gauge because a) there is an obvious operator formalism, and b) the string field is a gauge invariant (physical) observable. The light-front gauge string field may be decomposed [1] [2]

$$
\begin{gathered}
\Phi\left(x^{+}, x_{0}^{-}, \vec{x}(\sigma)\right)=\int_{-\infty}^{\infty} \frac{d \vec{p}}{(2 \pi)^{d-2}} \int_{0}^{\infty} \frac{d p^{+}}{2 p^{+}} \prod_{\ell=1}^{\infty} \sum_{\left\{\vec{n}_{\ell}\right\}} \\
\left(\mathbf{A}_{p^{+}, \vec{p},\left\{\vec{n}_{\ell}\right\}} e^{\left[i\left(p^{+} x_{0}^{-}+p^{-} x^{+}+\vec{p} \cdot \vec{x}_{0}\right)\right]} \mathrm{H}_{\vec{n}_{\ell}}\left(\vec{x}_{\ell}\right)+\text { h.c. }\right) .
\end{gathered}
$$

In other words, the string gauge invariance is fixed by the condition $X^{+}(\sigma)=x^{+}$, the other longitudinal string coordinate being (classically) determined by the reparametrization constraint

$$
X^{-}(\sigma)=x_{0}^{-}+\int^{\sigma} d \tilde{\sigma} \vec{X}^{\prime} \cdot \vec{P}(\tilde{\sigma})
$$

the transverse coordinates are expanded

$$
\vec{X}(\sigma)=\vec{x}_{0}+\sum_{\ell=1}^{\infty}\left(\vec{x}_{\ell} e^{i \ell \sigma}+\vec{x}_{\ell}^{*} e^{-i \ell \sigma}\right)
$$

(i.e. $x_{-\ell}=x_{\ell}^{*}$; for open strings, $x_{-\ell}=x_{\ell}=x_{\ell}^{*}$ ). The string creation/annihilation operators obey the canonical commutation relations

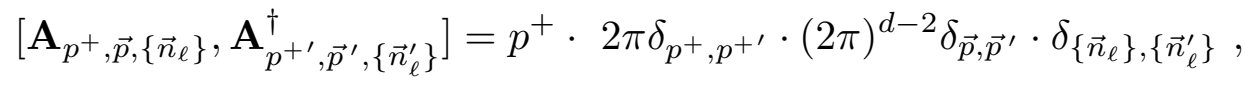

and the $\mathrm{H}_{n}$ are the harmonic oscillator wavefunctions of the string transverse normal modes. The mass shell condition is

$$
0=2 p^{+} p^{-}-\vec{p}^{2}-\sum_{\vec{n}_{\ell}=0}^{\infty} \ell \vec{n}_{\ell}-m_{0}^{2}
$$

Strictly speaking, of course, the ground state mass $m_{0}^{2}<0$ for the bosonic string and our integral expressions will be badly behaved. We will treat this intercept of the leading Regge trajectory as a free parameter to be adjusted according to our wishes; if one is a stickler, one can repeat the calculation in the superstring - the physics is unchanged (even there we will want to keep $m_{0}^{2}$ small and positive to separate the poles in the propagator, and tend it to zero only at the end of the calculation). 
Once again it is straightforward to evaluate the field commutator:

$$
\begin{aligned}
& {\left[\Phi\left(x^{+}, x_{0}^{-}, \vec{x}_{\ell}, \vec{x}_{\ell}^{*}\right), \Phi\left(x^{+^{\prime}}, x_{0}^{-\prime}, \vec{x}^{\prime}, \vec{x}_{\ell}^{* \prime}\right)\right] }=\int_{0}^{\infty} \frac{d p^{+}}{2 p^{+}} \\
&\left(\mathbf{K}_{p^{+}}^{0}\left(\delta x^{+}, \delta x_{0}^{-}, \delta \vec{x}\right) \prod_{\ell=1}^{\infty} \mathbf{K}_{p^{+}}^{\ell}\left(\delta x^{+}, \vec{x}_{\ell}, \vec{x}_{\ell}^{\prime}\right)-\right. \\
&\left.\mathbf{K}_{p^{+}}^{0}\left(-\delta x^{+},-\delta x_{0}^{-},-\delta \vec{x}\right) \prod_{\ell=1}^{\infty} \mathbf{K}_{p^{+}}^{\ell}\left(-\delta x^{+},-\vec{x}_{\ell},-\vec{x}_{\ell}^{\prime}\right)\right) .
\end{aligned}
$$

The $\mathbf{K}_{p^{+}}^{\ell}$ are harmonic oscillator propagators for the normal modes

$$
\begin{aligned}
\mathbf{K}_{p^{+}}^{\ell}\left(\delta x^{+}, \vec{x}_{\ell}, \vec{x}_{\ell}{ }^{\prime}\right) & =\left[\frac{i \ell}{\pi \sin \left[\left(\ell \delta x^{+}\right) / p^{+}\right]}\right]^{(d-2) / 2} \\
\times & \exp \left[\frac{-i \ell}{\sin \left[\left(\ell \delta x^{+}\right) / p^{+}\right]}\left(\left(\left|\vec{x}_{\ell}\right|^{2}+\left|\vec{x}_{\ell}^{\prime}\right|^{2}\right) \cos \left[\left(\ell \delta x^{+}\right) / p^{+}\right]-2 \operatorname{Re}\left(\vec{x}_{\ell}^{*} \cdot \vec{x}_{\ell}^{\prime}\right)\right)\right]
\end{aligned}
$$

Now the question arises, what are the analyticity properties of the integrand in the complex $p^{+}$plane? For $\mathbf{K}_{p^{+}}^{0}$, the answer is as before; for $\mathbf{K}_{p^{+}}^{\ell}$ one can continue the integral to the imaginary $p^{+}$axis as

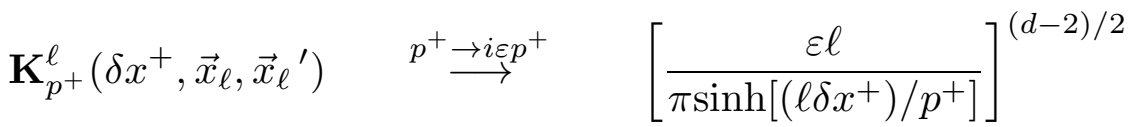

$$
\begin{aligned}
& \times \exp \left[\frac{-\varepsilon \ell}{\sinh \left[\left(\ell \delta x^{+}\right) / p^{+}\right]}\left(\left(\left|\vec{x}_{\ell}\right|^{2}+\left|\vec{x}_{\ell}^{\prime}\right|^{2}\right) \cosh \left[\left(\ell \delta x^{+}\right) / p^{+}\right]-2 \operatorname{Re}\left(\vec{x}_{\ell}^{*} \cdot \vec{x}_{\ell}^{\prime}\right)\right)\right] .
\end{aligned}
$$

The continued contribution to the integrand coming from the oscillators is regular as $p^{+} \rightarrow 0$; as $p^{+} \rightarrow \infty$, the exponent becomes

$$
-\frac{\varepsilon p^{+}}{\delta x^{+}}\left|\vec{x}_{\ell}-\vec{x}_{\ell}^{\prime}\right|^{2}+O\left(\frac{\ell \delta x^{+}}{p^{+}}\right)
$$

The $\operatorname{sign} \varepsilon= \pm 1$ is chosen according to the sign of $\delta x^{+}$so that the exponential is damped. Combining this result with the zero-mode propagator yields the condition for convergence of the analytically continued $p^{+}$integral:

$$
2 \delta x^{+} \delta x_{0}^{-}-\delta \vec{x}_{0}^{2}-\sum_{\ell=1}^{\infty}\left|\delta \vec{x}_{\ell}\right|^{2}<0 .
$$

The total propagator kernel will be continuable, and hence the two contributions to the commutator will cancel, provided this condition is satisfied. Otherwise, if the LHS is 
positive, one cannot continue the integral and the two measurements of the string field will interfere.

Unfortunately, in the string light-front gauge we find a physical string field at the expense of knowing where the string is in the full loop space. Eq.(1.5) says that, if we write the string field in position representation (as we must to look for the light cone), its transverse momentum is maximally uncertain and so is $X^{-}(\sigma)$. One might try to evade this by going to minimal uncertainty wavepackets for the string oscillator modes, but this is only achieved at the expense of smearing out the string field over the transverse space. We still lose control over the spacetime location of the strings.

Nevertheless there is a natural covariant generalization of the causal boundary, or string light cone (the vanishing locus of the LHS of (1.13)); namely

$$
0=\sum_{\ell=-\infty}^{\infty} \delta x_{-\ell}^{\mu} \delta x_{\mu \ell}=\int d \sigma \delta X^{2}(\sigma)
$$

The restriction of this expression to the light-front gauge $X^{+}(\sigma)=x^{+}$is precisely the string light cone determined above. It suffers from the problem that, while invariant under simulataneous reparametrizations of the two loops $X, X^{\prime}$, it is not invariant under independent reparametrizations of them. However this perhaps should not be expected given the above-mentioned reparametrization properties of the light-front gauge causal boundary. Eq.(1.14) has the appealing property that it is Lorentz covariant, in fact it is invariant under the entire spacetime conformal group, i.e. Lorentz transformations generated by $\int d \sigma X^{[\mu} P^{\nu]}$, dilations generated by $\int d \sigma X \cdot P(\sigma)$, and so on. In addition it has invariance under rotating the mode amplitudes among one another, which might be of relevance in the mythical $\alpha^{\prime} \rightarrow 0$ limit of string theory.

\subsection{Covariant Gauge}

In fact there is an ansatz in the covariant (Feynman-Siegel) gauge quantization which indeed reproduces the light cone (1.14). In field theory, the commutator of two fields can be expressed purely in terms of the Green's function

$$
\left\langle 0\left|\left[\phi(x), \phi\left(x^{\prime}\right)\right]\right| 0\right\rangle=\oint_{\mathcal{C}} \frac{d p^{0}}{2 \pi} \int \frac{d \vec{p}}{(2 \pi)^{d-1}} \frac{i e^{i k \cdot x}}{p^{2}-m^{2}}
$$

where from now on $\vec{x}, \vec{p}$ will denote $d-1$ component spatial quantities. All the standard

Green's functions arise from different choices of contour $\mathcal{C}$ in the complex $p^{0}$ plane; the 
commutator arises from the closed contour that surrounds both poles in the integrand. Consider this contour to be the difference of two contours, one slightly above and one slightly below the real $p^{0}$ axis. Writing the kernel in the Schwinger representation and doing the $p$ integrals, we find

$$
\left\langle 0\left|\left[\phi(x), \phi\left(x^{\prime}\right)\right]\right| 0\right\rangle=\int_{0}^{\infty} d \tau\left(\frac{1}{4 \pi \tau}\right)^{d / 2}\left(\exp i\left[\frac{\left(x-x^{\prime}\right)^{2}}{4 \tau}-m^{2} \tau\right]-\quad \text { h.c. }\right) .
$$

In other words we have the same expression as in light-front quantization with the lightfront momentum $p^{+}$replaced by the inverse of the Schwinger proper time $\tau$. The analyticity properties in the complex $\tau$ plane are thus determined by the sign of $x^{2}$, and as before (1.15) vanishes outside the light cone.

If we adopt (1.15) as a definition of the string field commutator in covariant quantization, then even though we have no proper spacetime operator formalism in covariant string field theory we can evaluate the commutator of two string fields (for an evaluation of the relevant world sheet path integrals, see e.g. [3]

$$
\begin{aligned}
& {\left[\Phi(X(\sigma), B(\sigma), C(\sigma)), \Phi\left(X^{\prime}\left(\sigma^{\prime}\right), B^{\prime}\left(\sigma^{\prime}\right), C^{\prime}\left(\sigma^{\prime}\right)\right)\right]=} \\
& \quad \int_{0}^{\infty} d \tau\left(\mathbf{K}_{\tau}^{0}\left(\delta x_{0}^{\mu}\right) \prod_{\ell=1}^{\infty} \mathbf{K}_{\tau}^{\ell}\left(x_{\ell}^{\mu}, x_{\ell}^{\mu^{\prime}}\right)-\mathbf{K}_{-\tau}^{0}\left(\delta x_{0}^{\mu}\right) \prod_{\ell=1}^{\infty} \mathbf{K}_{-\tau}^{\ell}\left(x_{\ell}^{\mu}, x_{\ell}^{\mu^{\prime}}\right)\right) .
\end{aligned}
$$

with $K_{\tau}^{0}\left(\delta x_{0}^{\mu}\right)$ the integrand in $(1.16)$ and

$$
\begin{array}{r}
\mathbf{K}_{\tau}^{\ell}\left(x_{\ell}^{\mu}, x_{\ell}^{\mu \prime}\right)=\left[\frac{i \ell}{\pi \sin (\ell \tau)}\right]^{(d-2) / 2} \\
\times \exp \left[\frac { - i } { \operatorname { s i n } ( \ell \tau ) } \left(\left(\ell\left|x_{\ell}\right|^{2}+\ell\left|x_{\ell}^{\prime}\right|^{2}+\left(c_{\ell} b_{\ell}^{*}+c_{\ell}^{*} b_{\ell}\right)+\left(c_{\ell}^{\prime} b_{\ell}^{* \prime}+c_{\ell}^{* \prime} b_{\ell}^{\prime}\right)\right) \cos (\ell \tau)\right.\right. \\
\left.\left.\quad-2 \operatorname{Re}\left(\ell x_{\ell}^{*} \cdot x_{\ell}^{\prime}\right)-\left(c_{\ell} b_{\ell}^{* \prime}+c_{\ell}^{*} b_{\ell}^{\prime}\right)-\left(c_{\ell}^{\prime} b_{\ell}^{*}+c_{\ell}^{* \prime} b_{\ell}\right)\right)\right],
\end{array}
$$

with $B(\sigma)=\sum b_{\ell} e^{i \ell \sigma}$ and $C(\sigma)=\sum c_{\ell} e^{i \ell \sigma}$; we have suppressed the ghost mode dependence in the arguments of (1.18) for convenience. Exactly the same reasoning used above in the analysis of the light-front gauge commutator leads to the conclusion that the causal boundary in covariant quantization is

$$
\begin{aligned}
0 & =\delta x_{0}^{2}+\sum_{\ell=1}^{\infty}\left(\delta x_{\ell} \cdot \delta x_{\ell}^{*}+\frac{1}{\ell}\left(\delta c_{\ell} \delta b_{\ell}^{*}+\delta c_{\ell}^{*} \delta b_{\ell}\right)\right) \\
& =\int d \sigma\left(\delta X^{2}(\sigma)+\delta C(\sigma) \frac{1}{\partial} \delta B(\sigma)\right)
\end{aligned}
$$

The restriction of this expression to the bosonic submanifold of the BRST superspace $\{X, B, C\}$ is indeed (1.14). 


\subsection{Consequences}

An interesting issue concerns the relation between the causal boundary (1.14) and the propagation of string mass eigenstates. To pass from the position representation $\Phi\left(x_{0},\left\{x_{\ell}\right\}\right)$ to mass eigenstates $\Phi\left(x_{0},\left\{n_{\ell}\right\}\right)$ one smears the former against harmonic oscillator wavefunctions $H_{n_{\ell}}\left(x_{\ell}\right)$. This entails an uncertainty $\Delta x_{\ell} \sim 1 / \ell$ and the string wanders logarithmically over all spacetime when all modes are considered. Clearly some renormalized concept of string location is needed. In any case causality for such smeared objects needs to be formulated.

The surprising thing about (1.14),(1.19) is that the causal region of string propagation is not the interior of the light cone of the underlying point manifold (defined by the set of pointlike loops). In other words, the region of causal contact of a string is NOT the union of the interiors of the point manifold light cones of the points along the string. Consider the case where one loop is pointlike at the origin and the other has zero extent in $X^{0} \equiv t$. Then Eq.(1.14) says that the string fields at these arguments interfere if the spatial sphere bounded by the radius of gyration $R_{\mathrm{gyr}}^{2}=\sum_{1}^{\infty}\left|x_{\ell}\right|^{2}$ of the future string obeys $t^{2}-r^{2} \geq R_{\mathrm{gyr}}^{2}$ ( $r$ is the radial coordinate of the center of mass of the second string). But this restriction does not forbid much of the string from being outside this light cone; much of the sphere of radius $R_{\text {gyr }}$ centered at $r$ may lie outside the point light cone (since $\left.\left(r+R_{\text {gyr }}\right)^{2} \geq r^{2}+R_{\text {gyr }}^{2}\right)$.

Thus Eq.(1.14) could prove of tremendous importance. Since strings interact by joining and splitting, one can imagine a process by which a piece of a string causally propagates outside the point light cone of a region of the point spacetime; that part of the string can then split off and influence physics in what to a pointlike observer is the spacelike region, yet string causality is obeyed. Actually this point is confusing. The split off string does not satisfy the causality condition with respect to the original string. A more conservative possibility is that the information propagated is still carried by the part of the string inside the point light cone. This would be the case for instance if the interference of the two orders of measurement is the same for the set of all strings which differ only outside one another's point light cone. Yet another outcome would be that the split off string still does not interfere with the original string unless it is inside the latter's string light cone; then it would still not be completely in the point spacelike region. Which of these possibilities is correct is tested when one calculates the commutator in the presence of a string vertex.

If (1.14) does reflect the causal structure of string theory, one must ask if there are black hole solutions to string theory having a horizon in the string sense, since the interior 
of an ordinary (point spacetime) black hole might in principle be probed by doing sensitive interference measurements with strings. Even if wavepackets cannot communicate across point horizons, could there be more subtle effects - for instance, scalar hair (which has profound influence on the horizon structure)? The extension of the analysis to the interacting theory should prove interesting.

Acknowledgements: It is a pleasure to thank J. Harvey, D. Kutasov, and P. Windey for discussions. 


\section{References}

[1] M. Kaku and K. Kikkawa, Phys. Rev. D10 (1974) 1110; Phys. Rev. D10 (1974) 1823.

See also J.F.L. Hopkinson, R.W.Tucker, and P.A. Collins, Phys. Rev. D12 (1975) 1653; E. Cremmer and J.-L. Gervais, Nucl. Phys. B90 (1975) 410.

[2] P. Goddard, J. Goldstone, C. Rebbi, and C. Thorn, Nucl. Phys. B56 (1973) 109; S. Mandelstam, Nucl. Phys. B64 (1973) 205.

[3] C. Ordóñez, M. Rubin, and R. Zucchini, Phys. Lett. 215B (1988) 103;

D. Birmingham and C.G. Torre, Phys. Lett. 205B (1988) 289. 